Check for updates

Cite this: RSC Adv., 2018, 8, 10163

Received 29th December 2017

Accepted 19th February 2018

DOI: 10.1039/c7ra13726e

rsc.li/rsc-advances

\section{Shunaoxin dropping pill, a Chinese herb compound preparation, attenuates memory impairment in D- galactose-induced aging mice}

\author{
Hong Zhou,,$^{\mathrm{ab}}$ Zhuo Qu,,$^{\mathrm{a}}$ Jingze Zhang, ${ }^{\mathrm{c}}$ Bingjie Jiang, ${ }^{\mathrm{a}}$ Changxiao Liu ${ }^{\mathrm{d}}$ \\ and Wenyuan Gao (iD *a
}

The Shunaoxin dropping pill (SNX) is derived from a traditional recipe. It has been used to treat cerebrovascular diseases in China since 2005 (approval number Z20050041). In this study, we used an in vitro $\mathrm{H}_{2} \mathrm{O}_{2}$-induced $\mathrm{PC} 12$ cell oxidative damage model and an in vivo D-gal-induced mouse memory impairment model to investigate whether SNX had neuroprotective effects. In vitro, prior to exposure to $100 \mu \mathrm{M} \mathrm{H}_{2} \mathrm{O}_{2}$ for $2 \mathrm{~h}$, PC12 cells were pre-treated with SNX $50 \mu \mathrm{g} \mathrm{mL} \mathrm{L}^{-1}$ for $24 \mathrm{~h}$. Hoechst 33258 staining was used to confirm the effect of SNX on apoptosis in the PC12 cells. Our results demonstrate that $\mathrm{H}_{2} \mathrm{O}_{2}$ suppresses the proliferation of PC12 cells and induces cell death. Pretreatment with SNX attenuates $\mathrm{H}_{2} \mathrm{O}_{2}$-induced apoptosis in PC12 cells. In vivo, D-gal was administered $\left(100 \mathrm{mg} \mathrm{kg}^{-1}\right.$, subcutaneously (s.c.)) once daily for 8 weeks to induce memory deficit and neurotoxicity in the brain of an aging mouse. Then, SNX (320 mg kg-1) was simultaneously administered orally. The present study demonstrates that SNX can alleviate aging in the mouse brain induced by D-gal via improving behavioral performance, alleviating oxidative stress, inhibiting neuroinflammation, and reducing brain cell damage in the hippocampus. Overall, these data clearly demonstrate the neuroprotective effect of SNX from the in vitro and in vivo results. SNX may be considered a novel agent for easing aging and/or age-related neurodegenerative diseases.

\section{Introduction}

Alzheimer's disease (AD), the most common type of age-related dementia, is characterized by memory deterioration and behavior disorder. The World Alzheimer Report 2016 estimated that there were 46.8 million people worldwide living with dementia in 2015 and this number will reach 131.5 million in 2050. Histopathological features of AD include the presence of extracellular deposits of $\beta$-amyloid peptides (plaques) and intracellular aggregates of the microtubule binding protein tau (tangles), which form the two distinguishing hallmark pathological features of $\mathrm{AD} .{ }^{1}$ Moreover, one of the strongest risk factors for the $\mathrm{AD}$ is brain aging. ${ }^{2}$ Reactive oxygen species (ROS)

${ }^{a}$ Tianjin Key Laboratory for Modern Drug Delivery and High-Efficiency, School of Pharmaceutical Science and Technology, Tianjin University, Weijin Road, Tianjin 300072, China. E-mail: pharmgao@tju,edu.cn; Fax: +86-22-87401895; Tel: +86-2287401895

${ }^{b}$ No. 6 Traditional Chinese Medicine Factory, Tianjin Zhongxin Pharmaceutical Group Corporation Ltd., Tianjin 300401, China

'Department of Pharmacy, Tianjin Key Laboratory of Cardiovascular Remodeling and Target Organ Injury, Logistics University of Chinese People's Armed Police Forces, Tianjin 300162, China

${ }^{d}$ The State Key Laboratories of Pharmacodynamics and Pharmacokinetics, Tianjin 300193, China

$\dagger$ These two authors contributed equally to this work. and oxidative stress are known to be associated with several ageassociated neuronal disorders. D-galactose (D-gal) is a physiological nutrient; however, accumulated $\mathrm{D}$-gal in vivo is metabolized by D-gal oxidases, which is accompanied by $\mathrm{H}_{2} \mathrm{O}_{2}$ generation, resulting in an increased oxidative damage. ${ }^{3}$ Moreover, a growing body of evidence indicates that D-gal overdose in animals causes neurotoxicity including oxidative stress, neuronal apoptosis, and astrocyte activation. ${ }^{4}$ Therefore, injection of D-gal is a model for brain aging, which induces and accelerates senescence in rodents to develop AD-like symptoms. ${ }^{5}$

The Shunaoxin dropping pill (SNX) is derived from a traditional recipe, which is composed of 2 herbs: Chuanxiong (Ligusticum chuanxiong Hort, Umbelliferae) and Danggui (Angelica sinensis radix, Umbelliferae). Both Chuanxiong and Danggui have been widely used in China, Japan, Korea, and other Asian countries for centuries. SNX is produced by supercritical fluid extraction as a solid dispersion and has been used to treat cerebrovascular diseases in China since 2005 (approval number Z20050041). Our previous research demonstrated that the six major chemical compounds in SNX extract were ferulic acid, senkyunolide $\mathrm{H}$, senkyunolide I, senkyunolide A, ligustilide, and levistolide A. ${ }^{6}$ Moreover, our previous results suggested that ligustilide-rich total lactones derived from the Shunaoxin dropping pill could alleviate the cognition decline triggered by 
diabetes. ${ }^{7}$ The reported studies showed that ligustilide exerted neuroprotective effects against ischemia/reperfusion-induced brain injury by minimizing oxidative stress and antiapoptosis. ${ }^{8,9}$ Ligustilide inhibits A $\beta 1-40$ toxicity on PC12 cells and ameliorates memory impairment induced by scopolamine in mice. ${ }^{10}$ This compound could be developed into a therapeutic agent to prevent and treat ischaemic disorders. In addition, ferulic acid inhibits A $\beta 1-40$ toxicity on PC12 cells, provides neuroprotection against oxidative stress-related apoptosis after cerebral ischemia/reperfusion injury in rats, and ameliorates memory impairment in a D-gal-induced aging mouse model. ${ }^{\mathbf{1 1 , 1 2}}$ Therefore, we suspect that SNX has a neuroprotective effect. In this study, we used an in vitro $\mathrm{H}_{2} \mathrm{O}_{2}$-induced PC12 cell oxidative damage model and an in vivo D-gal-induced mouse memory impairment model to investigate whether SNX had neuroprotective effects.

\section{Materials and methods}

\section{Materials}

SNX was obtained from Zhongxin Pharmaceuticals (Tianjin, China). D-gal and vitamin E (VE) were purchased from YiFang Technology Co., Ltd. (Tianjin, China). Donepezil (DON) was purchased from a drug store (Tianjin, China). Malondialdehyde (MDA), superoxide dismutase (SOD), glutathione (GSH), and glutathione peroxidase (GPx) assay kits were obtained from Nanjing Jiancheng Bioengineering Research Institute (Nanjing, China). Anti-cleaved caspase-3, anti-nuclear factor $\kappa \mathrm{B}(\mathrm{NF}-\kappa \mathrm{B})$, and anti-glial fibrillary acidic protein (GFAP) antibodies were purchased from Boster Biological Engineering Co., Ltd. (Wuhan, China). Biotinylated goat anti-rabbit secondary antibody and 3,3'-diaminobenzidine tetrahydrochloride (DAB) were purchased from ZSGB-BIO (Beijing, China). The other reagents were commercially available and of analytical purity.

\section{Neuroprotective effects of $\mathrm{SNX}$ on $\mathrm{H}_{2} \mathrm{O}_{2}$-induced cytotoxicity}

Cell culture. PC12 cells, a rat cell line derived from pheochromocytoma cells, were acquired from the Shanghai Institutes for Biological Sciences, Chinese Academy of Sciences (Shanghai, China), and were maintained on tissue culture plastic in an RPMI-1640 medium supplemented with $10 \%$ fetal bovine serum at $37{ }^{\circ} \mathrm{C}$ under an atmosphere of $5 \% \mathrm{CO}_{2}$ and $95 \%$ air.

Cell viability assay. Cell proliferation was measured by the colorimetric 3-(4,5-dimethylthiazol-2-yl)-2,5-diphenyltetrazolium bromide (MTT) assay. The amount of formazan was measured at $570 \mathrm{~nm}$ using a 96-well plate reader (BioTek Instruments, Inc.). Briefly, the cells were seeded at a density of $5 \times 10^{5}$ per well in the 96-well plates. Prior to exposure to $100 \mu \mathrm{M} \mathrm{H}_{2} \mathrm{O}_{2}$ for $2 \mathrm{~h}$, PC12 cells were pre-treated with SNX $\left(50 \mu \mathrm{g} \mathrm{mL}^{-1}\right)$ for $24 \mathrm{~h}$. After this, $200 \mu \mathrm{M}$ vitamin $\mathrm{E}$ was used as a positive control. Then, a fresh solution of MTT $\left(0.5 \mathrm{mg} \mathrm{mL} \mathrm{mL}^{-1}\right)$ was added to each well with further incubation for $4 \mathrm{~h}$. Finally, the cells were dissolved with $100 \mu \mathrm{L}$ of DMSO and then analyzed in a multiwell plate reader at $570 \mathrm{~nm}$.
Nuclear staining for the assessment of apoptosis. Chromosomal condensation and morphological changes in the nucleus of PC12 cells were observed using the chromatin dye Hoechst 33258. The PC12 cells were fixed with 100\% ice-cold methyl alcohol for $10 \mathrm{~min}$. After washing with phosphate buffer saline (PBS) three times, the nuclei were stained with $5 \mu \mathrm{g} \mathrm{mL}{ }^{-1}$ of Hoechst 33258 for $15 \mathrm{~min}$ and then observed using a fluorescence microscope equipped with UV filters.

\section{Neuroprotective effects of SNX on D-gal-induced impairment of memory and learning of aging mice}

Animal and drug administration. Kunming mice were obtained from the Experimental Animal Center, Chinese Academy of Medical Sciences, Peking, SCXK-2012-0004. The animals were housed in cages at $22 \pm 2{ }^{\circ} \mathrm{C}$ and a humidity of $40 \pm 5 \%$ under a $12 \mathrm{~h}$ light/dark cycle and received a standard diet and water ad libitum. The Animal Ethics Committees of the Faculty of Medicine approved all the experimental protocols in accordance with the Principles of Laboratory Animal Care and Use in Research (Ministry of Health, Beijing, China). After one week of acclimatization, all mice were screened by the Morris water maze test. The criteria for selecting qualified mice are the time of searching the hidden platform within a $1.5 \mathrm{~min}$ limit. Herein, 48 qualified mice were randomly divided into 4 groups of 12 each: control group (control), D-gal alone (D-gal), D-gal + DON $750 \mathrm{mg} \mathrm{kg}{ }^{-1} \mathrm{~d}^{-1}$, and D-gal + SNX $320 \mathrm{mg} \mathrm{kg}^{-1} \mathrm{~d}^{-1}$. SNX and DON were suspended in a carboxymethylcellulose sodium (CMC-Na) aqueous solution. The CMC-Na aqueous solutiontreated mice were used as the control group. SNX, DON or vehicle were administered by intragastric administration once daily for 8 weeks. Moreover, the mice were injected subcutaneously with D-gal in a dose of $100 \mathrm{mg} \mathrm{kg}^{-1} \mathrm{~d}^{-1}$ or normal saline (injected with an equal volume of saline for the control group) consecutively for 8 weeks (Fig. 1).

Morris water maze test. After the SNX treatment, mice $(n=12$ each group) were subjected to the Morris water maze test as described previously. ${ }^{13}$ Briefly, the experimental apparatus consisted of a circular pool filled with water $\left(23 \pm 1{ }^{\circ} \mathrm{C}\right)$. A platform was located $1 \mathrm{~cm}$ below the water surface at the center of the target quadrant, providing the only escape from water. During 6 days of training, the mice underwent 4 trials a day, alternating among 4 pseudorandom starting points. If a mouse failed to find the platform within $90 \mathrm{~s}$, it was guided to the platform by the researcher and kept there for $15 \mathrm{~s}$. Probe trials were conducted $24 \mathrm{~h}$ after the last training trial. During the probe trials, the platform was removed, and the mice were free to swim in the tank for $90 \mathrm{~s}$. The training and probe trials were recorded by a video camera mounted on the ceiling, and data were analyzed using the behavior analyzing system (Anhui Zhenghua Biological Equipment Co., Ltd, Anhui, China). This system was used to record the swimming trace and calculate the latency to the platform and platform crossing times. The number of times that the mice crossed the position where the platform was placed during the learning session (crossing times) and the time required to locate the hidden escape platform (escape latency) were determined using the behavior analyzing system. 


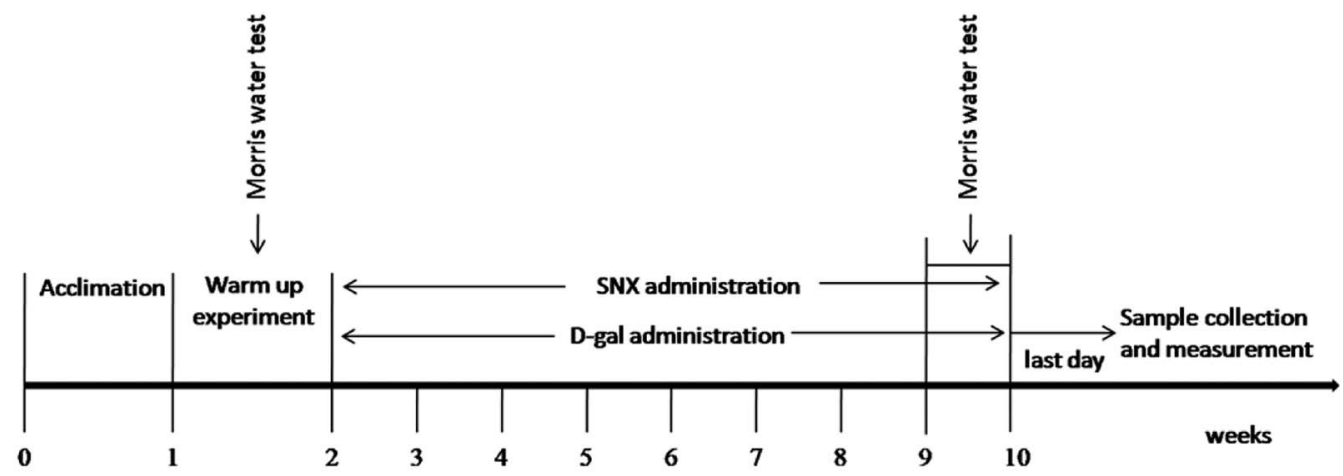

Fig. 1 The sequence of study procedures. After one week of acclimatization, all mice were screened by the Morris water maze test. Mice were injected subcutaneously with D-gal (100 mg kg $\mathrm{d}^{-1}$ for D-gal, D-gal plus SNX group) or normal saline (injected with an equal volume of saline for control group) consecutively for 8 weeks. Morris water maze test was performed at the 8 th week. After behavioral tests, mice were sacrificed, and brain tissues were obtained for biochemical analysis or histological examination.

Immunohistochemistry. For immunohistochemistry, endogenous peroxidase activity in the sectioned tissues was blocked with $3 \% \mathrm{H}_{2} \mathrm{O}_{2}$, and nonspecific binding sites were blocked with $3 \%$ normal goat serum (ZSGB-BIO, Beijing, China). After blocking, the sectioned tissues were incubated overnight in rabbit anticleaved caspase-3, anti-NF- $\mathrm{B}$, and anti-GFAP antibodies (Boster Biological Engineering Co., Ltd. Wuhan, China) diluted 1 : 100 in the blocking solution. Following incubation with the primary antibody, the sections were incubated for $1 \mathrm{~h}$ in biotinylated goat anti-rabbit secondary antibody diluted 1:500 in PBS and subsequently incubated with an avidin-biotin-horseradish peroxidase complex for $15 \mathrm{~min}$ at $37{ }^{\circ} \mathrm{C}$. The sections were washed twice with PBS and incubated in DAB, and then, the sections were washed with distilled water. The staining of caspase-3, NF- $\kappa \mathrm{B}$, and GFAP proteins were measured using the Image Pro Plus software (IPP 6.0, Media Cybernetics). Brown staining on the cell membrane or in the cytoplasm represented positive staining, and the staining density indicated the expression levels of caspase- 3 , NF- $\mathrm{B}$, and GFAP proteins.

Histopathological analysis. Brain tissues from each group were fixed in a $4 \%$ paraformaldehyde solution for $24 \mathrm{~h}$. After routine tissue processing, the tissues were embedded in paraffin. Then, $4 \mu \mathrm{m}$-thick sections obtained from each paraffin block were stained with hematoxylin and eosin (HE) and Nissl staining for histopathological evaluation. Images were obtained using a digital camera (ECLIPSE TS 100, Nikon) and analyzed using IPP 6.0.

Statistical analysis. All values are expressed as means \pm standard errors (s.e.). Data were analyzed by one-way analysis of variance (ANOVA), and differences among means were analyzed using the Dunnett's test or Fisher's Protected LSD multiple comparison test. Tests were performed using the SPSS 20.0 system (Chicago, IL); a $P$ value less than or equal to 0.05 was considered to be statistically significant.

\section{Results}

SNX improved the viability of PC12 cells damaged by $\mathrm{H}_{2} \mathrm{O}_{2}$

To verify the cytotoxic and neuroprotective effects of SNX for $\mathrm{H}_{2} \mathrm{O}_{2}$-induced PC12 cell oxidative injury, a cell viability assay was performed by the MTT method. The results indicate that SNX has no significant cytotoxicity (data not shown herein). As Fig. 2 illustrates, the cell viability was markedly decreased in PC12 cells damaged by $\mathrm{H}_{2} \mathrm{O}_{2}$, whereas SNX effectively protected the cells as compared to those in the model.

\section{SNX prevented apoptosis in PC12 cells induced by $\mathrm{H}_{2} \mathrm{O}_{2}$}

We observed the effect of SNX on the morphology of PC12 cells treated with $\mathrm{H}_{2} \mathrm{O}_{2}$. The PC12 cells in the control group grew well and maintained normal cell morphology (Fig. 3A). However, exposure to $\mathrm{H}_{2} \mathrm{O}_{2}$ for $2 \mathrm{~h}$ resulted in an obvious reduction in the number of viable cells and cell body shrinkage and disruption of the dendritic networks (Fig. 3A). However, cells in cultures pre-treated with SNX displayed normal morphology without remarkable cell number reduction and cell congregation.

The morphological changes were further confirmed using the DNA fluorescent dye Hoechst 33258. As illustrated in Fig. 3B, cells in the control group exhibited a high rate of healthy nuclei. However, cells treated with $\mathrm{H}_{2} \mathrm{O}_{2}$ alone contained a lot of small bright blue dots, representing chromatin condensation or nuclear fragmentation. Pretreatment with SNX could reverse these abnormalities, and a significant decrease in nuclear condensation and fragmentation was observed.

\section{SNX improved learning and memory of D-gal treated mice}

To investigate the effect of D-gal and SNX on mice behavior and memory function, the Morris water maze test was performed. As shown in Fig. 4A, the escape latency declined progressively during the 6 training days. Moreover, the escape latency in the D-gal group was much longer than that in the other groups for all training days. These results revealed that the D-gal-treated mice had significant memory impairment. After the trial session on day 7 , the hidden platform was removed, and the probe test was performed. As illustrated in Fig. $4 \mathrm{~B}$ and $\mathrm{C}$, the number of platform crossings and the time spent in the target quadrant were significantly increased by SNX treatment in the $\mathrm{D}^{-}$ gal-treated mice as compared to those in the case of the D-gal- 


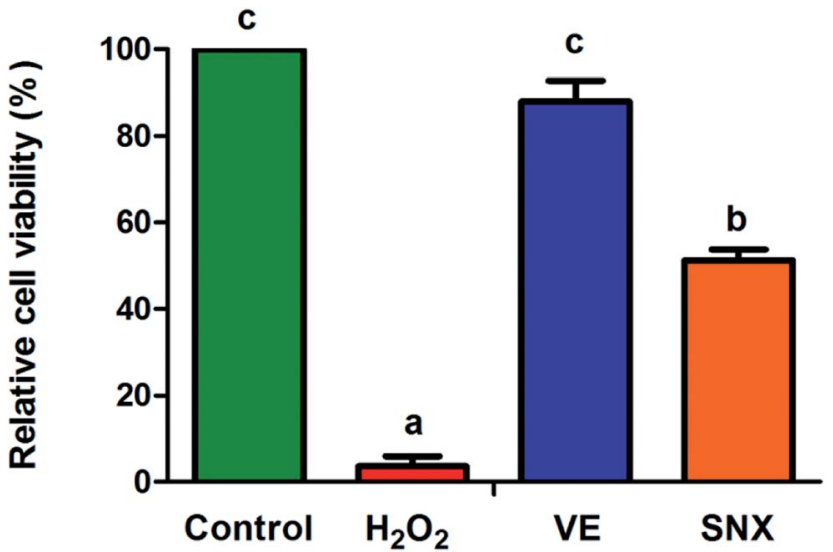

Fig. 2 PC12 cells were pre-treated with SNX $\left(50 \mu \mathrm{g} \mathrm{mL}^{-1}\right)$ for $24 \mathrm{~h}$. Thereafter, cells were treated with $100 \mu \mathrm{M} \mathrm{H}_{2} \mathrm{O}_{2}$ for $2 \mathrm{~h}$, and cell viability was determined by the MTT assay. The results are presented as mean \pm standard errors $(n=3)$. Different letters represent significantly different values as assessed by one-way ANOVA and LSD tests with $P<0.05$.

alone-treated mice. Compared with the vehicle control group, Dgal-alone-treated mice spent less time $(P<0.05)$ in the target quadrant and crossed to the platform fewer times $(P<0.05)$. Interestingly, there was a significant difference between the SNX-treated groups and the D-gal-alone-treated group, indicating that SNX could restore the D-gal-induced deficit of memory and learning ability. In addition, the swimming speed of the mice in the model group was lower than that in mice in the other groups, but there was no significant difference (data was not presented).

\section{SNX alleviated oxidative stress in the brain of D-gal-treated mice}

We have further examined several key antioxidants, including the enzymatic antioxidants SOD and GPx, which can scavenge ROS, GSH levels in the brain of D-gal-induced mice, and the oxidative stress-induced MDA levels. As illustrated in Table 1, compared to the control group, mice treated with D-gal showed a significant decrease in the content of GSH, activities of SOD, and GPx, whereas the MDA level was significantly increased $(P<$ 0.05). Administration of SNX resulted in antioxidant effects in $D^{-}$ gal-treated mice, as evidenced by an increase in the GSH content, SOD and GPx activities along with a decline of the MDA level in D-gal-alone-treated mice.

\section{SNX attenuated the caspase-3 protein expression in the hippocampus of $\mathrm{D}$-gal-treated mice}

To further investigate the neuroprotective mechanism of SNX, we observed the effect of SNX on the expression of caspase-3 protein in the CA1 region of the hippocampus through immunohistochemistry. The results shown in Fig. 5 demonstrated that D-gal treatment induced an increase in the activation of caspase-3 in the CA1 region of the hippocampus that was reversed by SNX, and a decrease in the number of active caspase-3-positive cells as compared to that in the D-gal treated group was observed $(P<0.05)$.

\section{SNX reduced the expression of the activated NF- $\kappa B$ in the brain of D-gal-treated mice}

The previous study demonstrated that D-gal activated NF- $\kappa$ B that led to neuroinflammation. According to the present immunohistochemistry analysis, the hippocampus NF- $\kappa \mathrm{B}$ expression in the $\mathrm{D}-$
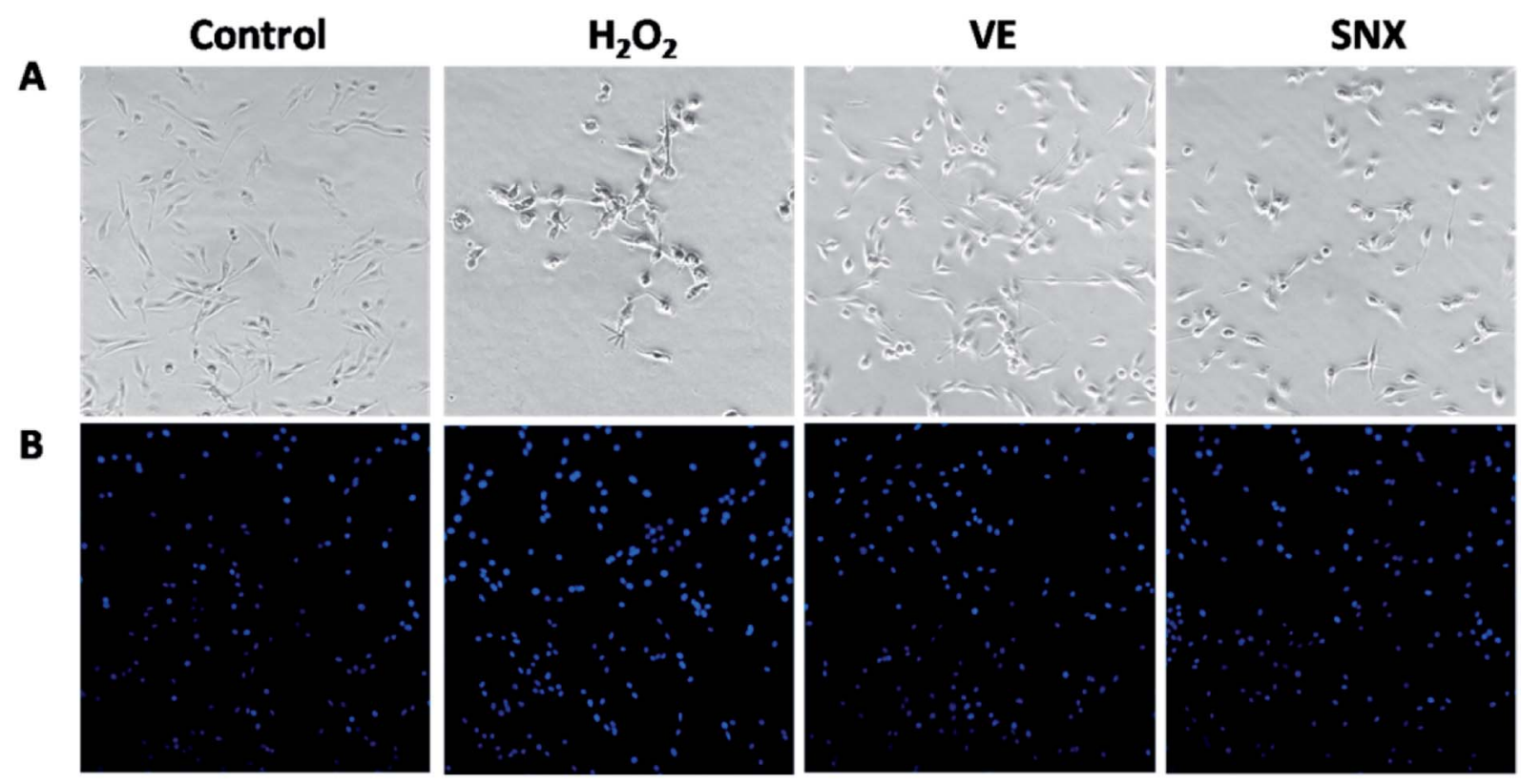

Fig. 3 Effects of SNX on cell morphology of $\mathrm{H}_{2} \mathrm{O}_{2}$-treated PC12 cells. SNX was added to the culture and incubated for $24 \mathrm{~h}$ before exposure to $100 \mu \mathrm{M} \mathrm{H}_{2} \mathrm{O}_{2}$ for $2 \mathrm{~h}$. Vitamin $\mathrm{E}(200 \mu \mathrm{M})$ served as a positive control. (A) Effect of SNX on $\mathrm{H}_{2} \mathrm{O}_{2}$-induced morphological changes in PC12 cells. (B) Morphological changes of PC12 cells observed by a fluorescence microscope after staining with Hoechst 33258. 
A

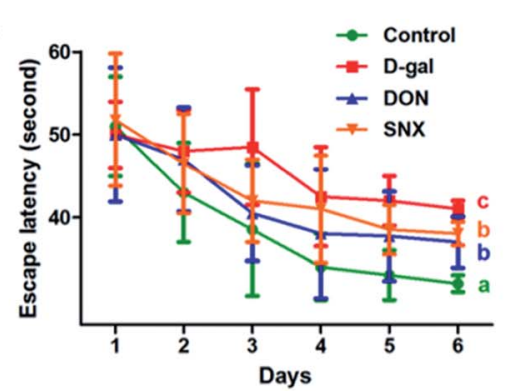

B

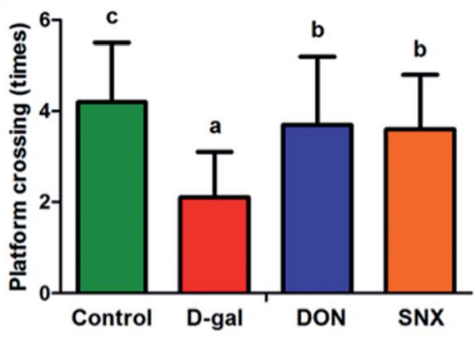

C ฐิ

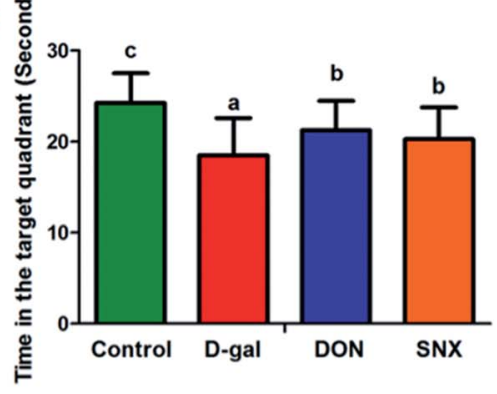

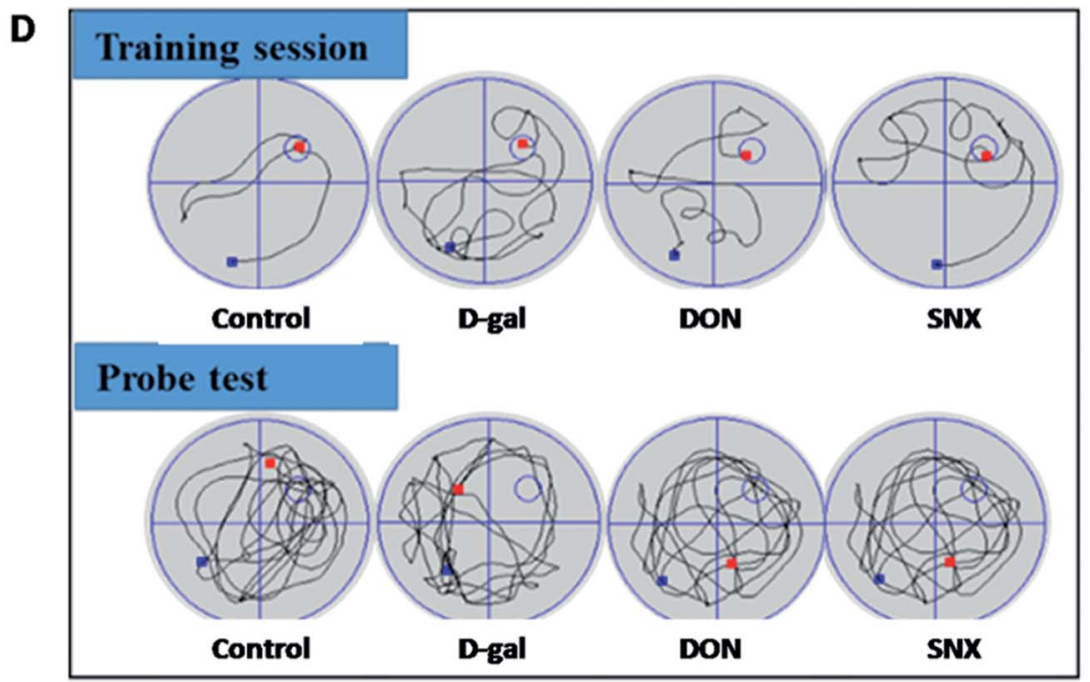

Fig. 4 Effect of SNX on memory impairment caused by D-gal administration ( $n=12$ each group). The Morris water maze test was carried out to test the spatial learning and memory ability of mice. (A) Latencies to find a hidden platform in the water maze during the 6 training days. (B) The number of crossing over the exact location of the previous platform during the probe test. (C) Time spent in the target quadrant (where the platform was located during training days) during the probe test. (D) The representative traces on the last day of training session and probe test. The results are presented as mean \pm standard errors $(n=12)$. Different letters represent significantly different values as assessed by one-way ANOVA and LSD tests with $P<0.05$.

gal-alone-treated group was significantly higher than that in the other group $(P<0.05)$. D-gal induced the increase of NF- $\mathrm{KB}$ expression in the hippocampus that was markedly restored by SNX administration $(P<0.05$, Fig. 5). These results suggest that the antiamnesic effects of SNX may be due to the inhibition of the NF-KB signaling pathway in the hippocampus.

\section{SNX inhibited the activation of astrocytes in the cortex of $\mathrm{D}^{-}$ gal-treated mice}

To analyze the expression of GFAP, we performed an immunohistochemistry technique. Our results shown in Fig. 5 indicated that the mice in the D-gal-alone-treated group had obviously more GFAP levels in the cortex than those in the control group. SNX significantly reduced the number of GFAP immunoreactive astrocytes from $45.8 \pm 3.2 \%$ in the cortex of D-gal-treated mice to $34.5 \pm 3.0 \%$, whereas DON (positive control) reduced the same to $36.3 \pm 2.7 \%$.

\section{Histopathological examination}

Next, we investigated the frontal brain atrophy in D-gal mice. As shown in Fig. 6, the frontal portion of the cerebrum showed atrophy-prone changes in D-gal mice. Administration with DON

Table 1 Effect of SNX on oxidative and anti-oxidant parameters in D-gal-induced aging mice ${ }^{a}$

\begin{tabular}{lclll}
\hline Groups & MDA $\left(\mathrm{nM} \mathrm{mg}^{-1}\right.$ protein) & GSH $\left(\mathrm{mg} \mathrm{g}^{-1}\right.$ protein $)$ & GPx $\left(\mathrm{mU} \mathrm{mg}^{-1} \text { protein }\right)^{\text {SOD }_{(\mathrm{U} \mathrm{mg}}{ }^{-1} \text { protein }^{-}}$ \\
\hline Control & $85.33 \pm 10.51 \mathrm{a}$ & $29.31 \pm 2.18 \mathrm{c}$ & $16.41 \pm 2.01 \mathrm{~b}$ & $0.81 \pm 0.07 \mathrm{~b}$ \\
D-gal & $117.67 \pm 11.39 \mathrm{c}$ & $15.44 \pm 1.98 \mathrm{a}$ & $11.59 \pm 1.99 \mathrm{a}$ & $0.67 \pm 0.05 \mathrm{a}$ \\
DON & $90.38 \pm 9.81 \mathrm{~b}$ & $23.45 \pm 3.27 \mathrm{~b}$ & $14.37 \pm 2.78 \mathrm{~b}$ & $0.77 \pm 0.05 \mathrm{~b}$ \\
SNX & $99.29 \pm 12.67 \mathrm{~b}$ & $22.76 \pm 3.12 \mathrm{~b}$ & $15.44 \pm 1.88 \mathrm{~b}$ & $0.75 \pm 0.03 \mathrm{~b}$
\end{tabular}

${ }^{a}$ The results are presented as mean \pm standard errors $(n=6)$. Different letters represent significantly different values as assessed by One-way ANOVA and LSD tests with $P<0.05$. 


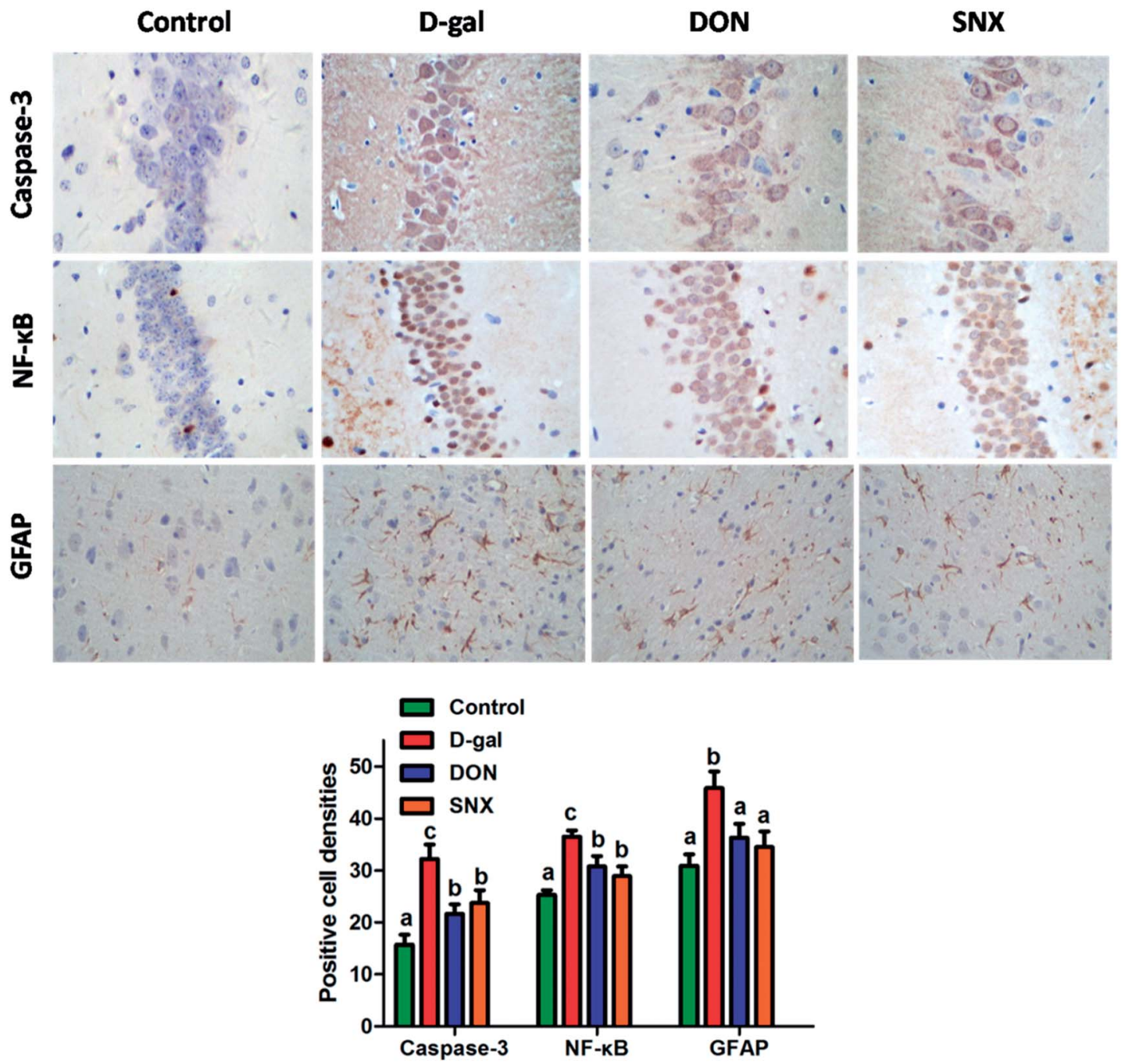

Fig. 5 Effect of SNX on the changes in brain weight and frontal portion of the cerebrum of D-gal induced memory impaired mice. The results are presented as mean \pm standard errors $(n=6)$. Different letters represent significantly different values as assessed by one-way ANOVA and LSD tests with $P<0.05$.

and SNX could alleviate the frontal brain atrophy induced by Dgal treatment. Moreover, we observed that the brain weight in the D-gal-alone-treated group decreased. However, there was no significant difference among all the groups. Histological features of the CA1 region of mice hippocampus are depicted in Fig. 7. There are no remarkable hippocampal neuronal abnormalities in the control group. CA1 pyramidal cells were arranged neatly and tightly with no noticeable cell loss. Compared with the case of the control group, D-gal induced obvious neuropathological changes in the CA1 region of the hippocampus, and extensively damaged neurons in the hippocampus CA1 region were observed. The CA1 layer neurons showed pronounced shrinkage of their neuronal bodies with their nuclei losing regular outlines and becoming hyperchromatic. However, SNX administration significantly attenuated the severity of neuronal pathomorphological changes. These data indicate that SNX protects the brain against D-gal induced injury, similar to the effects of DON.

\section{Nissl staining}

Nissl body staining is a marker of mature neurons and can be used to detect neuronal apoptosis. As illustrated in Fig. 7, the Nissl-stained slices of mouse brain showed intense, rich Nissl bodies in the control group, with clearly depicted axons and no obvious abnormalities in neurons. In the D-gal-alone-treated group, Nissl bodies were significantly reduced in the region of the hippocampus CA1. Treatment with SNX protected the neurons from the D-gal-induced damage. The quantification of Nissl-positive cell densities in the hippocampus was determined, as shown in Fig. 7. The number of survival neurons in the CA1 region of the hippocampus was reduced in D-gal-treated mice as compared to that in the vehicle-treated mice. After SNX and DON treatment, the number of survival neurons significantly increased in the CA1 region of the hippocampus in SNXtreated mice as compared to that in the D-gal-alone-treated group. These findings indicate that SNX protects against neuronal apoptosis in the hippocampus of D-gal treated mice. 


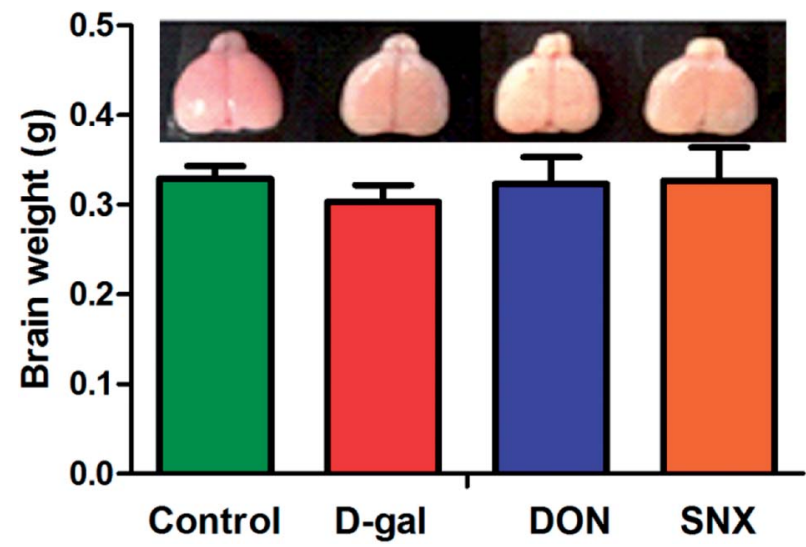

Fig. 6 Immunohistochemistry analysis of caspase- 3 and NF- $\kappa B$ in the CA1 region and GFAP in the cortex of mouse brain. Mean density of NF- $\kappa \mathrm{B}$, caspase-3, and GFAP were determined by Image Pro Plus 6 . The results are presented as mean \pm standard errors $(n=6)$. Different letters represent significantly different values as assessed by one-way ANOVA and LSD tests with $P<0.05$.

\section{Discussion}

During natural aging, the brain undergoes progressive morphologic and functional changes resulting in the observed behavioral retrogression such as decline in motor and cognitive performance. It will be of significant value to find out drugs against neurodegeneration to delay brain senescence. In this study, we demonstrated that SNX treatment protected the PC12 cells against $\mathrm{H}_{2} \mathrm{O}_{2}$-induced oxidative injury and apoptosis and the hippocampus against abnormalities using a wellcharacterized aging mouse model with D-gal administration. The results showed that SNX afforded significant cytoprotection against $\mathrm{H}_{2} \mathrm{O}_{2}$-induced apoptosis and delayed the senescence of the hippocampus induced by D-gal via anti-oxidation, antiinflammation, and anti-apoptosis.

At first, in vitro model system with $\mathrm{H}_{2} \mathrm{O}_{2}$-induced PC12 cell oxidative injury was used to illustrate the neuroprotective effect of SNX. It is widely accepted that oxidative stress can cause DNA damage. Hoechst 33258 staining was used to monitor the changes in the DNA and nuclear structure of $\mathrm{H}_{2} \mathrm{O}_{2}$-treated PC12 cells during growth in the presence or absence of SNX. Our present results demonstrate that $\mathrm{H}_{2} \mathrm{O}_{2}$ suppresses the proliferation of PC12 cells and induces cell death, as evidenced by Hoechst 33258 staining. Pretreatment with SNX attenuates $\mathrm{H}_{2} \mathrm{O}_{2}$-induced apoptosis in PC12 cells. We further carried out the in vivo experiments to confirm the neuroprotective effect of SNX.

D-gal is a reducing sugar and can be metabolized at normal concentrations. However, accumulated D-gal in vivo is metabolized by the D-gal oxidases accompanied by the generation of $\mathrm{H}_{2} \mathrm{O}_{2}$, resulting in the increase of oxidative damage. Mice administered with D-gal (100 $\mathrm{mg} \mathrm{kg}^{-1}$ intraperitoneally) for 60 days have neuroinflammation, elevation of ROS, and synaptic disorder. ${ }^{14}$ Therefore, we carried out this research to investigate the neuroprotective effect of SNX on the D-gal-induced aging mouse model from the perspective of anti-oxidation, antineuroinflammation, and anti-apoptosis.

Oxidative stress plays an important role in the aging process. ${ }^{15}$ MDA is a major marker of oxidative damage in cell

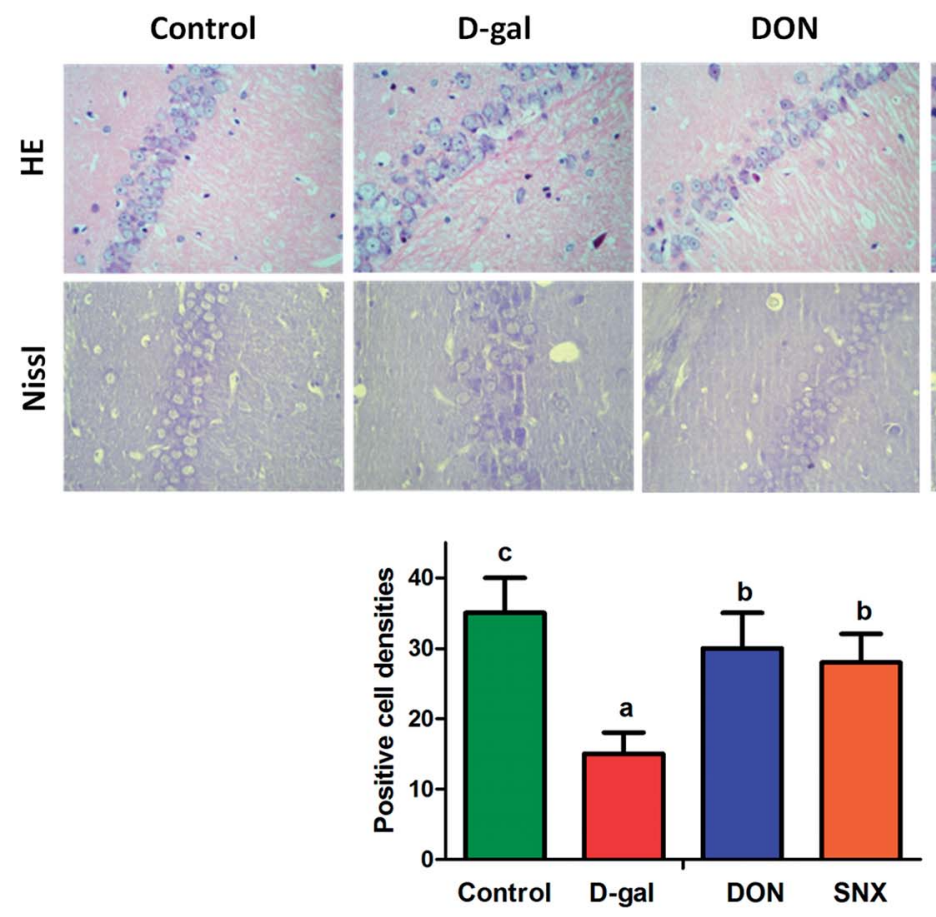

Fig. 7 Representative changes of the mice brain and photomicrograph of HE and Nissl staining in the CA1 region of hippocampus. Nissl-positive cell densities were determined by the Nissl staining in the hippocampus CA1 region of mice brain. The results are presented as mean \pm standard errors $(n=6)$. Different letters represent significantly different values as assessed by one-way ANOVA and LSD tests with $P<0.05$. 
membranes. SOD and GPx are the most important antioxidant enzymes that inhibit free radical formation. ${ }^{16}$ Mice treated with D-gal show decreased activities of SOD and GPx as well as decreased levels of GSH. ${ }^{17}$ In our present study, mice were given D-gal for 8 weeks. Brain MDA levels increased; however, SOD and GPx activities as well as GSH levels were found to decrease following D-gal treatment. These findings clearly show that D-gal treatment causes significant oxidative stress in the brain, as indicated by the increased MDA levels and diminished activity of the antioxidant enzymes. Interestingly, SNX could effectively restore these antioxidant enzymes and attenuate MDA production in the brain of D-gal mice; this suggested that SNX protected the mice against D-gal-induced brain oxidative stress by decreasing ROS and the lipid peroxide levels. These findings supported the view that aging was closely associated with an ROS attack, and SNX could obviously strengthen the antioxidative defense against free radicals and abate oxidative damage in the brain of D-gal-induced mice. However, further research is needed to reveal in greater detail the precise molecular mechanism of action.

Numerous mechanisms are associated with oxidative stressinduced neurodegeneration in the D-gal induced aging mouse model. ${ }^{18}$ Recent studies have reported that $\mathrm{D}$-gal administration involves cell apoptosis and caspase-3 activation in mice hippocampal neurons. ${ }^{19,20}$ To further investigate the possible mechanism of SNX in reversing D-gal induced memory decline, the level of caspases-3, one of the major apoptotic mediators, was examined. The present results demonstrate that treatment with SNX can reverse caspase-3 protein expression in the CA1 region, suggesting the anti-apoptotic capability of SNX.

Recent reports demonstrate that D-gal induces astrocyte dysfunction and inflammatory response. ${ }^{21}$ The increased GFAP expression in the cerebral cortex is an indicator of brain aging. $\mathrm{NF}-\kappa \mathrm{B}$, a family of DNA-binding proteins, is activated under physiological and pathological conditions and involved in regulating many aspects of cellular activity in stress, injury, and especially in pathways of inflammatory response. ${ }^{20}$ Therefore, in the present study, we observed the expression of GFAP in the cerebral cortex and $\mathrm{NF}-\kappa \mathrm{B}$ in the $\mathrm{CA} 1$ region. Interestingly, SNX attenuated a D-gal-mediated decrease in the astrocyte plasticity marker GFAP. The excessive increase in the GFAP expression resulting in astrogliosis implicates inflammation, increase in reactive oxygen species, and transition to a neurodegenerative state. ${ }^{22}$ Few studies propose the involvement of the transcription factors NF- $\kappa \mathrm{B}$ in regulation of the transcription of GFAP. In our present study, the results show that SNX prevents the D-galinduced memory impairment associated with the activation of NF- $\kappa$ B. SNX treatment significantly reduced the expression of $\mathrm{NF}-\kappa \mathrm{B}$ as compared to the case of the $\mathrm{D}$-gal administered group; this suggested that SNX could protect the hippocampus from age-induced chronic inflammation.

\section{Conclusion}

In general, the present study demonstrates the neuroprotective effect of SNX from the results of in vitro and in vivo experiments. SNX can alleviate aging in the mouse brain induced by D-gal by improving behavioral performance, alleviating oxidative stress, inhibiting neuroinflammation, and reducing brain cell damage in the hippocampus. SNX may be considered as a novel agent for easing aging and/or age-related neurodegenerative diseases.

\section{Conflicts of interest}

We have no conflict of interest in this research.

\section{Abbreviation}

$\begin{array}{ll}\text { AD } & \text { Alzheimer's disease } \\ \text { CMC-Na } & \text { Carboxymethylcellulose sodium } \\ \text { DAB } & 3,3^{\prime} \text {-Diaminobenzidine tetrahydrochloride } \\ \text { D-gal } & \text { D-galactose } \\ \text { DON } & \text { Donepezil } \\ \text { ROS } & \text { Reactive oxygen species } \\ \text { VE } & \text { Vitamin E } \\ \text { NF- } \kappa \text { B } & \text { Nuclear factor } \kappa \text { B } \\ \text { GFAP } & \text { Glial fibrillary acidic protein } \\ \text { PBS } & \text { Phosphate buffer saline } \\ \text { HE } & \text { Hematoxylin and eosin } \\ \text { MDA } & \text { Malondialdehyde } \\ \text { SNX } & \text { Shunaoxin dropping pill } \\ \text { SOD } & \text { Superoxide dismutase } \\ \text { GSH } & \text { Glutathione } \\ \text { GPx } & \text { Glutathione peroxidase }\end{array}$

\section{Acknowledgements}

The work was supported by the Special Financial Grant received from the China Postdoctoral Science Foundation (No. 2015T81140), PhD Research Startup Foundation of Logistics University of Chinese People's Armed Police Forces (No. WHB201509), and Science and Technology Support Program Foundation of Tianjin China (No. 15CZDSY01020).

\section{References}

1 S. W. Pimplikar, J. Clin. Immunol., 2014, 34, 64-69.

2 J. H. Zhu, X. Y. Mu, J. Zeng, C. Y. Xu, J. Liu, M. S. Zhang, C. P. Li, J. Chen, T. Y. Li and Y. P. Wang, PLoS One, 2014, 9, e101291.

3 A. F. Aydın, J. Çoban, I. Doğan-Ekici, E. Betül-Kalaz, S. DoğruAbbasoğlu and M. Uysal, Metab. Brain Dis., 2016, 31, 337345.

4 C. X. Chen, L. Y. Huang, Z. H. Nong, Y. X. Li, W. Chen, J. P. Huang, X. R. Pan, G. W. Wu and Y. Z. Lin, Neurochem. Res., 2017, 1-14.

5 T. Ali, H. Badshah, T. H. Kim and M. O. Kim, J. Pineal Res., 2015, 58, 71-85.

6 L. Huo, J. Z. Zhang, Z. Qu, H. Chen, Y. M. Li and W. Y. Gao, J. Ethnopharmacol., 2015, 173, 352-360.

7 H. Zhou, Z. Qu, J. Z. Zhang, Y. X. Liu, H. G. Yang, H. Chen, Y. M. Li, C. X. Liu and W. Y. Gao, RSC Adv., 2016, 6, 109132. 
8 X. M. Wu, Z. M. Qian, L. Zhu, F. Du, W. H. Yung, Q. Gong and Y. Ke, Br. J. Pharmacol., 2011, 164, 332-343.

9 H. Y. Peng, J. R. Du, G. Y. Zhang, X. Kuang, Y. X. Liu, Z. M. Qian and C. Y. Wang, Biol. Pharm. Bull., 2007, 30, 309-312.

10 L. L. Cheng, X. N. Chen, Y. Wang, L. Yu, X. Kuang, L. L. Wang, W. Yang and J. R. Du, Fitoterapia, 2011, 82, 1128-1132.

11 C. Y. Cheng, S. Y. Su, N. Y. Tang, T. Y. Ho, S. Y. Chiang and C. L. Hsieh, Brain Res., 2008, 1209, 136-150.

12 H. G. Yang, Z. Qu, J. Z. Zhang, L. Q. Huo, J. Gao and W. Y. Gao, Int. J. Food Sci. Nutr., 2016, 67, 806-817.

13 Z. Qu, H. G. Yang, J. Zhang, L. Q. Huo, H. Chen, Y. M. Li, C. X. Liu and W. Y. Gao, Neurochem. Res., 2016, 41, 21992214.

14 H. C. Huang, B. W. Zheng, Y. Guo, J. Zhao, J. Y. Zhao, X. W. Ma and Z. F. Jiang, J. Alzheimers Dis., 2016, 52, 899-911.
15 G. Paradies, G. Petrosillo, V. Paradies and F. M. Ruggiero, Neurochem. Int., 2011, 58, 447-457.

16 S. J. Tsai and M. C. Yin, Eur. J. Pharmacol., 2012, 689, 81-88. 17 Z. Qu, J. Z. Zhang, H. G. Yang, L. Q. Huo, J. Gao, H. Chen and W. Y. Gao, Physiol. Behav., 2016, 154, 114-125.

18 A. Kumar, A. Prakash and S. Dogra, Int. J. Alzheimer's Dis., 2011, 2011, 1-9.

19 X. Cui, P. P. Zuo, Q. Zhang, X. K. Li, Y. Z. Hu, J. G. Long, L. Packer and J. Liu, J. Neurosci. Res., 2006, 83, 1584-1590.

20 J. Lu, Y. L. Zheng, D. M. Wu, L. Luo, D. X. Sun and Q. Shan, Biochem. Pharmacol., 2007, 74, 1078-1090.

21 M. Lei, X. Hua, M. Xiao, J. Ding, Q. Han and G. Hu, Biochem. Biophys. Res. Commun., 2008, 369, 1082-1087.

22 E. C. Phillips, C. L. Croft, K. Kurbatskaya, M. J. O'Neill, M. L. Hutton, D. P. Hanger, C. J. Garwood and W. Noble, Biochem. Soc. Trans., 2014, 42, 1321-1325. 